\title{
Drugs that made headlines in 2014
}

This year's newsworthy drugs made major strides against infectious diseases, cancer and more. Some others received attention for controversies they stemmed or stomped. Here is a look at the therapies that leapt forward, some that are in limbo, and others that fell by the wayside.

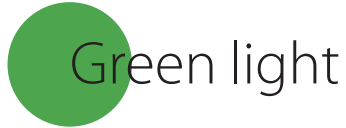

\section{Antifibrotic drugs}

The US Food and Drug Administration (FDA) approved two drugs to treat idiopathic pulmonary fibrosis (IPF) in October. Esbriet (pirfenidone), manufactured by InterMune and now a part of Switzerland-based Roche, and Ofev (nintedanib), from Germany-based Boehringer Ingelheim, act against pathways that promote tissue scarring, delaying the decline of lung function caused by IPF. But each of the drugs costs around $\$ 95,000$ per year of treatment, raising concerns that insurers may hesitate to cover treatment for milder forms of the disease.

\section{GSC-100}

La Jolla Pharmaceutical in San Diego, California, reported positive results this year for a new therapeutic option for people with chronic kidney disease. Its drug, GSC-100, works to block galectin-3, a protein whose overexpression has been linked to organ failure and cancer. Results released in March from an ongoing phase 2 trial showed that treatment with GSC-100 for eight weeks improved kidney function when compared to a placebo. An extension of the trial is underway to measure the effects of long-term dosing.

\section{LCZ696}

In August, Swiss drug maker Novartis published the results of a phase 3 trial of its investigational heart failure medicine, LCZ696. The drug combines valsartan and sacubitril to block both angiotensin receptors and a protein that contributes to vasoconstriction. In a trial involving over 8,000 patients, LCZ696 seemed to significantly reduce the rate of death from cardiovascular disease. $16.5 \%$ of patients with chronic heart failure on the angiotensinconverting enzyme inhibitor called enalapril died from cardiovascular disease during the study period, compared with $13.3 \%$ on LCZ696-a 20\% lower rate. The drug is now under priority review at the FDA.

\section{CYD-TDV}

Paris-based Sanofi Pasteur announced this fall that its dengue vaccine CYD-TDV was nearly $61 \%$ effective in preventing infection in a second phase 3 trial of more than 20,000 children. Protection against each of the four dengue serotypes ranged from $42 \%$ to $78 \%$, with serotypes 1 and 2 having the lowest protection. The vaccine also helped reduce hospitalizations due to dengue by $80 \%$. Sanofi Pasteur plans to have approval in time for a late 2015 market release.

\section{PD-1 blockers}

The FDA approved New Jersey-based Merck's Keytruda (pembrolizumab) in September for advanced melanomas that are resistant to other drugs. Keytruda is the first approved monoclonal antibody that blocks PD-1, a protein that dampens $\mathrm{T}$ cell responses. Opdivo (nivolumab), made by Bristol-Myers Squibb, also blocks PD-1 and is under priority review by the FDA as a breakthrough therapy for several cancers, including melanoma.

\section{Imbruvica}

In July, the FDA approved a breakthrough cancer therapy for chronic lymphocytic leukemia (CLL) by expanding the label of a currently available drug, Imbruvica (ibrutinib). The drug, which is manufactured by Sunnyvale, California-based Pharmacyclics and New Jersey-based Janssen Biotech, disrupts an overactive signaling pathway in malignant $B$ cells. In a phase 3 trial with a median follow-up of 9.4 months, Imbruvica showed $78 \%$ reduction in the risk of progression of the disease or death compared with those receiving another drug approved to treat CLL, ofatumumab.

\section{Impavido}

In March, the FDA approved the first drug to treat cutaneous or mucosal leishmaniasis, an infection caused by parasites transferred through sand fly bites that can cause a range of symptoms from difficulty breathing to skin sores. The amoeba-killing drug Impavido (miltefosine), made by Montreal-based Paladin Therapeutics, was also awarded a tropical disease priority review voucher by the FDA, which-upon payment of a fee-can be used towards a priority review for future products the company may develop. Impavido is also approved to treat visceral leishmaniasis, which affects internal organs.

\section{Belsomra}

Merck's Belsomra (suvorexant) won FDA approval in August for the treatment of insomnia. The drug works as an antagonist to receptors for orexin, a neurotransmitter that promotes wakefulness, whereas most available insomnia treatment pathways work to promote sleepiness.

\section{Entyvio}

In May, Takeda Pharmaceuticals' Entyvio (vedolizumab) was approved for patients with ulcerative colitis and those with Crohn's disease who are not benefitting from other drugs. By blocking adhesion molecules, the monoclonal antibody prevents a specific subset of $\mathrm{T}$ cells from localizing in the gut, where they cause chronic inflammation and exacerbate disease.

\section{T cell therapy}

Researchers at the University of Pennsylvania in Philadelphia, in collaboration with Novartis, published in October the results of a pilot study of $\mathrm{T}$ cell therapy for pediatric acute lymphoblastic leukemia. The therapy, which had received FDA breakthrough status in July, involves treating patients with their own $\mathrm{T}$ cells that have been genetically reprogrammed to target CD19, a protein expressed on leukemia cells. Ninety percent of the 30-patient study group went into complete remission despite having previous refractory or relapsing disease. Novartis will seek FDA approval in 2016.

\section{Obeticholic acid}

Obeticholic acid, produced by Intercept Pharmaceuticals for treatment of primary biliary cirrhosis, showed early promise in a phase 2 trial, driving up the company's stock prices. A later phase 3 study published in April showed that this bile acid analog reduces serum levels of a liver protein called alkaline phosphatase, which serves as a marker for organ damage. The drug then went on to receive a fast-track designation from the FDA in May.

\section{Harvoni}

Gilead combined its hepatitis C drug, Sovaldi (sofosbuvir) with a new antiviral called ledipasvir to generate Harvoni, the first FDAapproved combination treatment for hepatitis C. Harvoni was approved in October after 94-99\% of the 1,500 patients in three different phase 3 trials no longer had detectable hepatitis $\mathrm{C}$ virus levels in their blood. 


\section{FMT pills}

In an attempt to make fecal transplants more palatable than traditional methods that employ nasal tubes, researchers at Massachusetts General Hospital in Boston created capsules containing frozen fecal matter as an alternative to more expensive ways of performing a fecal matter transplant (FMT). The experimental capsules, aimed at treating recurring Clostridium difficile infections, cured 18 of the
20 people enrolled in the trial of symptoms such as diarrhea-demonstrating tremendous promise for FMT pills as a viable treatment option.

\section{Dalvance}

In May, the antibiotic Dalvance (dalbavancin), made by Durata Therapeutics, became the first qualified infectious disease product (QIDP) to gain FDA approval for the treatment of acute bacterial skin and skin structure infections (ABSSSIs). The QIDP designation is reserved for antibacterials and antifungals devised to treat life-threatening infections. In two clinical trials, Dalvance, which works by interrupting cell wall synthesis in ABBSSI-causing bacteria such as methicillin-resistant Staphylococcus aureus, cleared $90 \%$ of nearly 1,300 patients of their skin or soft tissue infections caused by S. aureus.

\section{Yellow light}

\section{Crenezumab}

In July, Roche announced that its drug, crenezumab, an antibody designed to clear the plaque-forming peptide beta amyloid, did not improve daily function or slow cognitive decline for patients with Alzheimer's disease in a phase 2 trial. A subset of patients with mild symptoms, however, experienced a $23.8 \%$ reduction in cognitive decline, encouraging Roche to move forward with a trial in patients with genetic susceptibility to Alzheimer's.

\section{PARP inhibitors}

Although they initially failed to improve outcomes for patients with ovarian cancer, poly-ADP-ribose polymerase (PARP) inhibitors may be making a comeback, specifically for patients with $B R C A$ mutations. AstraZeneca in London reported that its PARP inhibitor, olaparib, extended progression-free survival for this subset of patients. Although the results are promising, olaparib was denied accelerated approval in July; FDA approval awaits the results of an ongoing phase 3 trial.

\section{Afrezza}

The FDA approved the inhalable insulin formulation Afrezza under the agency's Risk Evaluation and Mitigation Strategy in June. The approval stipulates that the maker of Afrezza, MannKind Corporation, must relay the risk of acute bronchospasms to healthcare providers and test for other risks, including effects on pediatric patients. The drug is on track to be available in early 2015 .

\section{Anti-Ebola therapy}

In the wake of the largest Ebola outbreak in history, several companies are vying to be the first to come up with a viable therapy. ZMapp, the monoclonal antibody mixture made by Mapp Biopharmaceutical in San Diego, California, has not yet progressed beyond animal dosing trials or compassionate use in humans. The Bill \& Melinda Gates Foundation and the United States Army Medical Research Institute of Infectious Diseases are working to help increase ZMapp's availability. In Canada,
Tekmira Pharmaceuticals has been developing an RNA interference drug called TKM-Ebola, which was granted expanded access for patients with Ebola. North Carolina-based drug developer Chimerix is meanwhile testing the antiviral drug brincidofovir, which has been given to at least two US patients with Ebola in two phase 2 clinical trials and will be tested by the humanitarian group Doctors Without Borders in Guinea and Liberia.

\section{Translarna}

The first drug aimed at treating the underlying cause behind Duchenne muscular dystrophy (DMD) received a conditional approval from the European Medicines Agency (EMA) this year. Translarna promotes the production of normal dystophin protein in patients with nonsense mutations, which can instigate DMD. The maker of the drug, New Jerseybased PTC Therapeutics, reported that it slowed the decline of the patients' ability to walk. The EMA will allow PTC to provide Translarna to patients with DMD while the company completes a confirmatory phase 3 clinical trial.

\section{Red light}

\section{Albumin}

A study published in April by a group of Italian researchers put to rest a controversy over the safety and efficacy of albumin added to intravenous fluids as a regulator of blood pressure and inflammation in patients with severe sepsis. Even though daily albumin administration for 28 days was deemed safe, it did not result in reduced rates of organ failure, time in intensive care or number of deaths when compared to patients who received standard intravenous fluids alone.

\section{Darapladib}

In May, GlaxoSmithKline (GSK) reported that its 15,000-patient study on its atherosclerosis candidate, darapladib, failed to prevent cardiac events among patients with chronic coronary heart disease. Darapladib inhibits lipoprotein-associated phospholipase A2, an enzyme thought to exacerbate inflammation in atherosclerotic plaques.

\section{Neuraminidase inhibitors}

In April, the Cochrane Collaboration published a comprehensive review of the effects of neuraminidase inhibitors against influenza such as Roche's Tamiflu (oseltamivir) and GSK's Relenza (zanamivir). The review, which included 107 randomized placebo-controlled trials, indicated that neither Tamiflu nor Relenza reduced complications of flu infection. In a statement published on its website in April, Roche said that it "fundamentally disagrees" with Cochrane's conclusions about Tamiflu.

\section{Statins}

Two trials published in May failed to show that statins improve outcomes for patients with chronic obstructive pulmonary disease (COPD) or acute respiratory distress syndrome (ARDS). The prospective trials showed no effect of Merck's Zocor (simvastatin) for COPD or AstraZeneca's Crestor (rosuvastatin) for ARDS when compared to placebo.

\section{mGluR5 antagonists}

In September, Roche terminated its fragile $\mathrm{X}$ drug development program after two phase 2 trials failed to meet primary and secondary endpoints. The trials tested the safety and efficacy of the metabolic glutamate receptor 5 (mGluR5) antagonist RG7090. Novartis also saw negative results of phase 2 and 3 trials testing the mGluR5 antagonist mavoglurant (AFQ056).

Amanda Keener 